\title{
CONDICIONES CLAVE PARA EL ÉXITO Y SOSTENIBILIDAD DE LOS EMPRENDIMIENTOS SOLIDARIOS DE MEDELLIIN*
}

\author{
Olga Lucía Arboleda Álvarez** \\ Hernando Zabala Salazar***
}

Recibido: marzo 02 de 2010

Aceptado: enero 20 de 2011

\section{RESUMEN}

El objetivo del artículo es analizar condiciones clave de éxito en los emprendimientos solidarios de Medellín, observadas desde la complejidad de sus desarrollos. La metodología aplicada se inscribe en el enfoque cualitativo interpretativo, a partir del cual se trabaja con una muestra a criterio de seis emprendimientos solidarios de Medellín y sus corregimientos; estos son abordados mediante técnicas como la entrevista semiestructurada a informantes clave, que se complementa con la aplicación de encuestas a miembros de dichas formas asociativas; la información es analizada mediante construcción categorial y técnicas estadísticas descriptivas. El resultado establece que el éxito de estos emprendimientos está determinado por el acercamiento al cumplimiento del objeto social; por su capacidad de arraigarse en un territorio concreto; por operar como un proceso de encadenamientos de empresas de su mismo tipo y por el fomento de procesos autogestionarios y participativos.

\section{PALABRAS CLAVE}

Organización empresarial, emprendimientos solidarios, cultura corporativa, empresas cooperativas, trabajadores propietarios.

\section{CLASIFICACIÓN JEL} L22; L26; M14; P13; J54

\section{CONTENIDO}

Introducción; 1.Referentes conceptuales y teóricos para la identificación de los emprendimientos solidarios; 2.Aspectos característicos de las condiciones claves para el éxito de los emprendimientos solidarios en Medellín; 3.Conclusiones; Bibliografía.

\footnotetext{
Este artículo es resultado de la investigación "Eficacia en la gestión de los emprendimientos solidarios del municipio de Medellín en relación con los servicios y beneficios percibidos por sus grupos de interés", realizada en el 2009 por Olga Lucía Arboleda A. (Investigadora principal), Hernando Zabala Salazar (Coinvestigador) y Gladis Bedoya Restrepo (Auxiliar), adscritos al grupo de Economía Solidaria (ECOSOL), de la Fundación Universitaria Luis Amigó (FUNLAM) y escalafonado en la categoría C de Colciencias. La investigación fue financiada por la FUNLAM y ejecutada durante el año 2009.

.* Historiadora de la Universidad Nacional, Medellín, Colombia. Especialista en Docencia Investigativa Universitaria de la Fundación Universitaria Luis Amigó, Medellín, Colombia. Docente investigadora de la Facultad de Ciencias Administrativas, Económicas y Contables y Directora del grupo de investigación ECOSOL de la FUNLAM. Dirección: transversal 51A No 67 B 90, Tel 4487666 ext. 9550, Medellín, Colombia. Correo electrónico: oarboled@funlam.edu.co.

** Historiador de la Universidad de Antioquia, Medellín, Colombia. Especialista en Derecho Cooperativo y Solidario de la Universidad Cooperativa de Colombia, Medellín, Colombia. Docente de cátedra de la Facultad de Ciencias Administrativas, Económicas y Contables de la Fundación Universitaria Luis Amigó y miembro del grupo de investigación ECOSOL. Director de la Corporación para el Desarrollo de la Comunidad y la Cooperación-CDC-, Medellín, y asesor de CONFECOOP-ANTIOQUIA. Dirección: transversal 51 A No 67 B 90 , Tel 4487666 ext.9550, Medellín, Colombia. Correo electrónico:hzabala@funlam.edu.co
}

Semestre Económico, volumen 14, № 28, pp. 77-94 • ISSN 0120-6346, enero-junio de 2011, Medellín, Colombia 
KEY CONDITIONS FOR THE SUCCESS AND SUSTAINABILITY IN MEDELLIN'S SOLIDARITY ENTREPRENEURSHIP ABSTRACT

The objective of this article is to analyze the key success conditions for the solidarity entrepreneurship in Medellin, observed for the complexity of their developments. The applied methodology is based on a qualitative interpretative scope, working with a chosen sample of six solidarity entrepreneurships from Medellin city and its sub-divisions; these are approached by technics such as semi structured interviews and key reports which are complemented with surveys applied to members of such associative forms; the information is analyzed through a categorical construction and descriptive statistics technics. The result establishes that the success of entrepreneurships is determined by the approach the clear scope of complying with the social objective; for its capacity to settle in a concrete region; for operating as a chaining process with companies with a similar objective and for supporting the self-management and participative processes.

\section{KEYWORDS}

Enterprise organization, solidarity entrepreneurship, corporate structures, cooperative companies, workers owners.

JEL CLASSIFICATION

L22; L26; M14; P13; I54

\section{CONTENT}

Introduction; 1.theoretical and conceptual concepts for identifying solidarity entrepreneurships.; 2.Charctersitic aspects for key conditions that guarantee success in solidarity entrepreneurship in Medellin; 3.Concluisons; Bibliography.

\section{CONDIÇÕES CHAVE PARA O SUCESSO E A SUSTENTABILIDADE DOS EMPREENDIMENTOS SOLIDÁRIOS EM MEDELLÍN}

\section{RESUMO}

O objetivo do artigo é analisar condições chave do sucesso nos empreendimentos solidários de Medellín observando eles desde a complexidade de seus desenvolvimentos. A metodologia aplicada acolhe-se ao enfoque qualitativo interpretativo, a partir do qual se trabalha com uma mostra ao critério de seis empreendimentos solidários de Medellín e suas subdivisões; estes são abordados a traves de técnicas como a entrevista semiestruturada aos informantes chaves as quais são complementadas com a aplicação de pesquisas de opinião aos membros de estas formas associativas, a informação é analisada mediante a construção categorial e técnicas descritivas. O resultado estabelece que o sucesso destes empreendimentos estã determinado pela aproximação ao cumprimento do objetivo social; pela sua capacidade de se acoplar a um território concreto; por operar como um processo de encadeamentos de empresas de seu mesmo tipo e pelo fomento de processo auto regressivos e participativos.

\section{PALAVRAS-CHAVES}

Organização empresarial, empreendimentos solidários, cultura corporativa, empresas cooperativas, trabalhadores proprietários.

\section{CLASSIFICAÇÃO JEL}

L22; L26; M14; P13; J54

\section{CONTEÚDO}

Introdução; 1. Referentes conceptuais e teóricos para a identificação dos empreendimentos solidários; 2. Aspectos característicos das condições chave para o sucesso dos empreendimentos solidários em Medellín; Conclusões; Bibliografia. 


\section{INTRODUCCIÓN}

El tema de los emprendimientos encuentra un referente de contextualización en la Ley 1014 de enero de 2006 de fomento a la cultura del emprendimiento, donde se define el concepto como

... una manera de pensar y actuar orientada hacia la creación de riqueza. Es una forma de pensar, razonar y actuar centrada en las opor tunidades, planteada con visión global y llevada a cabo mediante un liderazgo equilibrado y la gestión de un riesgo calculado, su resultado es la creación de valor que beneficia a la empresa, la economía y la sociedad.

En dicha ley, pueden destacarse también los principios generales que regirán toda actividad de emprendimiento:

a) Formación integral en aspectos y valores como desarrollo del ser humano y su comunidad, autoestima, autonomía, sentido de pertenencia a la comunidad, trabajo en equipo, solidaridad, asociatividad y desarrollo del gusto por la innovación y estímulo a la investigación y aprendizaje permanente.

b) Fortalecimiento de procesos de trabajo asociativo y en equipo en torno a proyectos productivos con responsabilidad social.

c) Reconocimiento de la conciencia, el derecho y la responsabilidad del desarrollo de las personas como individuos y como integrantes de una comunidad.

d) Apoyo a procesos de emprendimiento sostenibles desde la perspectiva social, cultural, ambiental y regional.

Igualmente, el tema encuentra elementos de contextualización en la propuesta del DNP (2007, p. 23), que específicamente sobre el fomento del emprendimiento señala la intencionalidad de articularlos a cadenas de valor con potencial real de mercado, que permitan ampliar la base empresarial y generar cambios sustanciales en la estructura productiva nacional.

Ya ubicados en el contexto local, en la ciudad de Medellín, la Alcaldía de Medellín (2004 y 2008), en los dos últimos planes de desarrollo 2004-2007 y 2008-2011, hace expresas consideraciones orientadas a desarrollar los emprendimientos productivos sostenibles y auto-gestionados; por ello es importante identificar aquí los diferentes tipos de emprendimiento:

- Los de tipo tradicional, en los cuales las funciones de la empresa creada se orientan a fabricar o comercializar un producto pero con el interés de conseguir unas ganancias para sus socios, además, su tipo de gestión es vertical y los espacios de decisión se corresponden con las juntas directivas.

- Los diseñados para generar valor social, desde actividades relacionadas con los sectores de la producción, la oferta de servicios o la comercialización; adoptan diversas formas de organización en las que se priorizan la innovación, la autogestión, la cooperación y la participación para beneficio colectivo.

- Existe también el denominado emprendimiento verde, en el cual se hace uso inteligente de los recursos y se usa fuente de energía alternativa.

En general, los emprendimientos tienen la capacidad de combinar recursos y capacidades laborales, tecnológicas, organizativas y comerciales de carácter tradicional con otras surgidas de dinámicas económicas contemporáneas, para generar una variada y heterogénea cantidad de actividades productivas orientadas a asegurar la subsistencia y la vida cotidiana.

Entre el 2004 y el 2007, la ciudad vio nacer una estrategia encaminada a promover el desarrollo de una nueva cultura emprendedora: la "Cultura E". A partir de este acontecimiento, la Administración municipal reconoció que en el territorio habitan miles de emprendedores, diferenciados por sus condiciones socio-económicas: emprendedores barriales comunitarios, jóvenes emprendedores universitarios, emprendedores ubicados en corregimientos, en conjunción con una creciente motivación para producir investigaciones, surgidas en 
ámbitos académicos o de otro tipo, que contribuyen al emprendimiento.

En este sentido, "Cultura E" constituyó una serie de mecanismos que tienen aplicabilidad según el tipo de emprendedor; por ejemplo, el Banco de las Oportunidades se encargó de desarrollar instrumentos y estrategias de intervención como son los Centros de Desarrollo Empresarial Zonal (CEDEZOS), la Red de Microcrédito y el Concurso de Capital Semilla para ponerlos a disposición de los emprendedores barriales; la Secretaría de Desarrollo Social implementó el componente de presupuesto participativo, el cual ha dimensionado el empresarismo social y generado una alta posibilidad de emprendimiento comunitario; el Departamento Administrativo de Planeación estableció programas y proyectos como los Concursos Planes de Negocios para fomentar emprendimientos de valor agregado en estudiantes de educación superior, egresados, profesores e investigadores.

El fortalecimiento del programa "Cultura E" como la estrategia para el desarrollo empresarial de Medellín y el Valle de Aburrá ha buscado transformar en la población valores, actitudes y competencias, mediante acciones integrales, que incidan en todos los procesos de emprendimiento, desde el más sencillo hasta el más sofisticado, cubriendo los campos de educación, formación para el trabajo, apoyo a la creación de empresas, fortalecimiento del tejido empresarial existente, formalización empresarial, financiación y procesos de innovación.

En consecuencia con lo anterior, se considera pertinente precisar el concepto de emprendimiento solidario como aquella modalidad de organización económica y de trabajo, a la cual, y guiados por los principios de autogestión, cooperación, eficiencia y viabilidad, acuden los sujetos sociales que tienen como única herramienta su fuerza de trabajo. Dicha organización se manifiesta en forma de asociaciones, cooperativas o grupos de producción, en la perspectiva del logro de condiciones para la sobrevivencia humana (Gaiger, 2003, p. 229)
Teniendo como referencia estos antecedentes y el estudio realizado por la Fundación Universitaria Luis Amigó sobre características socioeconómicas y organizacionales de los emprendimientos solidarios en Medellín (Lopera, 2008), el objetivo principal de este artículo es presentar resultados sobre elementos, aspectos o condiciones que se consideran clave para el éxito de dichos emprendimientos, tanto desde el punto de vista de las influencias externas (entes gubernamentales u otras entidades nacionales e internacionales), como también y muy especialmente por sus propios desarrollos internos.

En cuanto a la metodología aplicada, debe decirse que esta se inscribe en el enfoque cualitativo interpretativo y es complementada con algunas técnicas empírico descriptivas, desde las cuales se procedió a abordar las diferentes experiencias de emprendimientos solidarios en Medellín y algunos de sus corregimientos, mediante la selección de una muestra no probabilística, a criterio de los investigadores, de seis experiencias de los emprendimientos referidos: Corporación Olas (COROLAS) del corregimiento de San Antonio de Prado; Precooperativa Multiactiva (COOPEUNO) del barrio Santo Domingo Savio de Medellín; Asociación de Recuperadores Prestadores de Servicios del corregimiento de Santa Elena (ARPSE); Asociación de Recuperadores Pioneros del corregimiento de Alta Vista (ARPA); Asociación Circuito Económico del corregimiento de San Antonio de Prado y Corporación Primavera del barrio Lovaina de Medellín.

En este orden de ideas para el trabajo de campo, en dichos contextos empresariales se tuvo la oportunidad de generar información mediante técnicas como la entrevista semiestructurada a informantes clave, el cuestionario y el diario de campo con registros de observación.

En cuanto al tratamiento de la información, con relación a lo cualitativo interpretativo se realiza un proceso de análisis mediante la identificación de categorías emergentes, provenientes de la información generada en los procesos de entrevista y 
observación. A partir de ello se definen y conceptualizan las categorías analíticas o definitivas. En lo empírico-descriptivo el plan de análisis contempló el vaciado de datos provenientes de los cuestionarios y la elaboración de tablas y gráficos de frecuencia derivados del análisis estadístico.

Con el análisis de los diferentes tipos de información (cuantitativa y cualitativa) se realiza un proceso de triangulación que permite aproximarse a la identificación y sustentación de las condiciones de algunos aspectos o elementos considerados claves para el éxito y sostenibilidad de los emprendimientos estudiados, inscritos en la racionalidad de la economía solidaria en la ciudad de Medellín.

Sobre la estructura del presente artículo, luego de esta introducción, se presentan algunos referentes teóricos y conceptuales para la identificación de los emprendimientos solidarios; luego en el nivel de los resultados, se exponen las condiciones para el éxito de los mencionados emprendimientos desde varios aspectos así: las acciones de autonomía y autodinamismo en relación con la intervención de los agentes externos que inciden en su desarrollo; la existencia y construcción de prácticas de autogestión, participación y productividad; y el efecto social producido por los emprendimientos en términos de capacidad de inserción socio laboral; el aumento en los niveles de ingreso; las condiciones de vida de los asociados y sus familias; el desarrollo de proyectos productivos; la construcción de trayectorias socioculturales manifestadas en el logro de arraigos locales y la construcción de redes o circuitos de cooperación mutua.

En las conclusiones se sintetizan los principales elementos reconocidos para la existencia de emprendimientos solidarios exitosos en el contexto local, los cuales están enmarcados en el logro de efectos sociales derivados del cumplimiento de su objeto social; el arraigo territorial; la construcción de circuitos de cooperación mutua y el fomento a procesos de autogestión y participación. En este orden de ideas se evidencia también la necesidad de una política pública con claves de intervención que garanticen continuidad en los procesos de apoyo a los emprendimientos.

\section{REFERENTES CONCEPTUALES Y TEÓRICOS PARA LA IDENTIFICACIÓN DE LOS EMPRENDIMIENTOS SOLIDARIOS}

La teoría del emprendimiento solidario surge del análisis realizado por Gide (1968, p. 50), quien resume la apuesta del emprendimiento social cooperativo así:

Cuando traté de representarme la organización de la sociedad futura, en la medida que nuestra ciencia, de no muy largas vistas, nos permite prever el porvenir, ella se me aparece bajo el aspecto de una multitud de asociaciones de todas clases y proporciones, las más inmensas, las otras pequeñas y de las cuales formarán parte libremente todos los hombres, fuera de algunos salvajes; asociaciones en las cuales los trabajadores percibirán el producto íntegro de su trabajo, porque ellos poseerán sus instrumentos de producción; asociaciones que suprimirán los intermediarios, porque ellos cambiarán sus productos directamente; asociaciones que no mutilarán el individuo porque la iniciativa individual se conservará como el resorte oculto que hará mover a cada una de ellas y que por el contrario protegerán al individuo contra los azares de la vida por la práctica de la solidaridad; asociaciones, en fin, que, sin suprimir la emulación que es indispensable al progreso, atenuarán la concurrencia y la lucha, suprimiendo la mayor parte de las causas que ponen en nuestra época a los hombres en conflicto.

Por su parte, en Latinoamérica sobresalen diversos autores que se han preocupado por el tema de las iniciativas económicas populares desde la perspectiva socioeconómica:

Guerra (2006) alude al término de emprendimientos solidarios para referirse a la experiencia que surge de la unión de las personas alrededor de una propuesta económica solidaria basada en relaciones de igualdad, democracia y solidaridad. 
Para Ortiz (2005) los emprendimientos mencionados están más próximos conceptualmente a la economía de trabajo, ejercida como medio de vida por los pobres para satisfacer sus necesidades básicas. En este esfuerzo, las familias campesinas y populares ponen continuamente sus capacidades de trabajo que puede ser remunerado en dinero en algunos casos. Los emprendimientos solidarios reconocidos como economía de trabajo permitirían una maximización de la capacidad productiva de las familias para realizar operaciones económicas de escala que no serían posible por otros medios, al tiempo que vivencian los valores comunitarios.

Razeto (1992), propone la "teoría económica comprensiva" para comprender las relaciones sociales y solidarias que se presentan en la economía solidaria que configuran una racionalidad diferente a la institucional del sector público y la de mercado correspondiente al sector privado. En tal sentido, los grupos humanos que se organizan lo pueden hacer bajo distintas lógicas que luego los caracterizan. La teoría propuesta por Razeto (1992) permite hacer lectura, no funcional, de otras racionalidades que involucran dimensiones que la teoría económica convencional no contempla.

En este sentido, es posible hacer lectura y comprender, al estilo Razetiano, el mundo de los pobres que no es solo fuente de conflicto social, sino también de desarrollo de solidaridad, lo que a menudo encuentra expresión en las distintas formas de economía solidaria.

Así, el concepto de emprendimiento más estrechamente ligado a la economía solidaria es propuesto por Razeto (1993, p. 24-26), quien desde la experiencias de la economía popular en Chile observó, en poblaciones de las periferias urbanas, grupos que emprendían organizaciones económicas, con recursos personales, puestos en común para ser trabajados desde acciones de ayuda mutua, solidaridad y cooperación, en la perspectiva de satisfacer necesidades de subsistencia, logrando incluso con algunos de ellos mejorar condiciones de calidad de vida y alcanzar niveles de acumulación y crecimiento económico.

La perspectiva de estas ideas emprendedoras encuentra también un elemento determinante en el factor trabajo en el cual según Razeto (1998, p. 4142) se expresa "la libre voluntad de una obra común... un trabajo que gratifica a las personas porque trabajan unidas, generando vinculos de amistad, solidaridad y comunidad".

Igualmente, Razeto (1998) identifica este fenómeno con un concepto que integra diferentes búsquedas de orden humano y no solo instintivo de conservación, para concretarlas en proyectos solidarios que articulan las dimensiones económica, social, política y cultural.

De este modo, la búsqueda por la subsistencia se integra con otras necesidades de orden axiológico y ontológico, en la perspectiva de una nueva concepción del desarrollo; aquí son pertinentes las reflexiones de Maxneef, Elizalde y Hopenhayn (1995, p. 51), quien argumentan:

Integrar la realización armónica de necesidades humanas en el proceso de desarrollo significa la oportunidad de que las personas puedan vivir ese desarrollo desde sus comienzos, dando origen así a un desarrollo sano, autodependiente y participativo, capaz de crear los fundamentos par un orden en el que se pueda conciliar el crecimiento económico, la solidaridad social y el crecimiento de las personas y de toda la persona.

En coherencia con estos referentes y en la perspectiva de la identificación de los emprendimientos solidarios debe decirse que estos adquieren unas características específicas que delimitan sus formas de actuación respecto de los demás tipos de emprendimiento (basados en la predominancia del factor capital o del factor gestión).

Existen multitud de propuestas en torno a la identificación de estos emprendimientos, pero casi todas ellas resaltan el hecho de que su fin último no es la maximización del factor capital ni la obtención de utilidades, sino lograr mejores beneficios para el 
mayor número de personas y de sus comunidades. Y como lo establece la Ley de Economía Solidaria en Colombia, que contribuyan al desarrollo ecológico, social y culturalmente sustentable.

Así, los aspectos que permiten diferenciar estas formas empresariales son sus competencias, lo cual implica:

a) Capacidad organizativa: esto es, que se estructure bajo un sistema de reglas autónomamente definidas y libremente aceptadas por los comprometidos, en las que se establezcan compromisos de participación (económica, social y cultural), los niveles de mando y de toma de decisiones y los mecanismos de cohesión social.

b) Capacidad empresarial: se trata de formas de asociación que han de cumplir la doble función de productoras de satisfactores (de bienes o servicios) y generadoras de ingresos (valores agregados a redistribuirse entre los factores).

c) Capacidad de impacto social: son formas de organización que se sustentan en la potenciación de los factores trabajo y comunidad y que, consecuentemente, redistribuyen la riqueza generada principalmente entre estos factores, contribuyendo a mejorar las condiciones de vida de los individuos y las colectividades.

d) Capacidad rentable: son formas empresariales que al potenciar otros factores productivos logran disminuir los costos de operación, eliminar la dependencia del capital y generar mayores valores agregados.

e) Capacidad integrativa: son formas empresariales que unen sus esfuerzos a las de otras formas empresariales (de naturaleza similar o por relación territorial o productiva), conformando redes que hacen posible la maximización colectiva de los factores.

Estas características generales del emprendimiento solidario instrumentalizan una última competencia: su capacidad de éxito por encima de la experiencia de crisis cíclicas que caracterizan los emprendimientos basados en los factores capital y gestión.

\section{ASPECTOS CARACTERISTICOS DE LAS CONDICIONES CLAVE PARA EL ÉXITO DE LOS EMPRENDIMIENTOS SOLIDARIOS EN MEDELLÍN}

Teniendo en cuenta el referente que permite entender los factores clave de éxito de las formas empresariales estudiadas como aquellas que explican sus fortalezas y buen desempeño básicamente en "relación al desarrollo de productos, el impacto social comunitario y la organización" (Gallo, 2005), los resultados se presentan en términos de las características de los elementos o aspectos que asumen una importancia máxima en los emprendimientos observados: la intervención de los agentes externos durante el proceso emprendedor; los temas de autogestión, participación y productividad; la proyección social que genera el emprendimiento y las relaciones de cooperación.

Lo anterior conlleva un análisis de las mencionadas formas empresariales desde la complejidad de sus desarrollos internos y de los resultados que en el tiempo producen.

\subsection{Intervención externa en la gestión de los emprendimientos solidarios de Medellín}

Cuando los emprendimientos solidarios están influenciados exclusivamente por agentes externos, estos últimos generalmente se limitan a una acción inicial facilitadora de la formación del proyecto emprendedor: hasta aquí llega su intervención y dejan que los grupos se desenvuelvan por sí mismos.

Esta situación debe analizarse muy especialmente en el caso de los emprendimientos de Medellín, sobre todo porque se ha observado, en los que fueron objeto de estudio, que la mayoría de ellos (60\%) tienen una fuerte influencia de agentes externos gubernamentales, en su concepción y constitución.

La política pública en estas materias a veces es discontinua, aunque en el caso de Medellín, en las últimas administraciones, se ha mantenido en los planes de desarrollo y en los presupuestos.

Por ejemplo, la Alcaldía de Medellín (2004, p. 127-140), en el Plan de Desarrollo 2004-2007, espe-

Semestre Económico, volumen 14, N²8, pp. 77-94 • ISSN 0120-6346, enero-junio de 2011, Medellín, Colombia 
cíficamente de la línea de trabajo No. 4, denominada Medellín productiva, competitiva y solidaria, generó una dinámica enfocada hacia el fortalecimiento de los emprendimientos productivos sostenibles y autogestionarios denominados emprendimientos solidarios.

A partir de este enfoque se consideró que la forma de generar inclusión económica y social era propiciar el desarrollo de propuestas de origen comunitario y carácter solidario, las cuales, como sostiene Razeto (1988) citado por Álvarez (2008, p.37) tienen la capacidad de

... combinar recursos y capacidades laborales, tecnológicas, organizativas y comerciales de carácter tradicional con otras surgidas de dinámicas económicas contemporáneas, para generar una variada y heterogénea cantidad de actividades productivas orientadas a asegurar la subsistencia.

Igualmente, la Alcaldía de Medellín (2008, p. 119), en la línea 3 del Plan de Desarrollo de Medellín 2008-2011, que trata sobre el desarrollo económico de la ciudad, expresa el propósito de:

Continuar consolidando el desarrollo humano integral y territorial, a partir del reconocimiento de la diversidad de su población, potenciar sus capacidades y capitales propios y articular, sin exclusión, las diferentes expresiones económico-empresariales que tienen presencia en todo el territorio, para hacer una ciudad más competitiva y solidaria; constituyéndose en una plataforma de oportunidades para el desarrollo empresarial individual y colectivo. En consecuencia, se promoverá la generación de fuentes de trabajo de calidad e ingresos, a partir de la apropiación de una cultura emprendedora y de desarrollo empresarial, la inserción en el mundo del trabajo mediante la formación en competencias laborales; el desarrollo de emprendimientos sociales con sentido solidario y el fomento de la asociatividad, que hagan a Medellín atractiva para la inversión nacional e internacional.
Aquí es importante también tener en cuenta algunas experiencias foráneas. Por ejemplo, a partir de los planteamientos de Fantova (1995, p. 60-62), se ha evaluado en el caso español sobre las fases que deben cumplir los agentes externos en el acompañamiento de los emprendimientos sociales:

- Primera: deben dar respuesta a las necesidades de capacitación y formación de los emprendedores, permitiéndoles construir su idea de negocio y generando un sentido de autonomía.

- Segunda: es necesario guiarles en la búsqueda de información y mercados; también en el descubrimiento de alternativas de financiamiento o en facilitar éste.

- Tercera: cuando se inicia el emprendimiento, el agente externo debe ayudarles a poner en marcha la empresa y colaborarles en formación de aptitudes específicas: gestión, ventas, trámites administrativos y legales, etc.

- Cuarta: avanzado el emprendimiento (dos o tres años) el interventor cambia su papel y se convierte en asesor y consejero, para contribuir a dar respuesta autónoma a las problemáticas del desarrollo.

- Quinta: en la etapa última de la intervención (a los cuatro o cinco años) suele suceder las crisis (generalmente por descontrol ante el éxito o el manejo inadecuado de las finanzas). Por eso el interventor asume el papel de consejero para ayudar a administrar el crecimiento y contribuir a producir cambios en el rumbo del emprendimiento.

Puede decirse que los emprendimientos en Medellín no manifiestan esta lógica. Las intervenciones son generalmente coyunturales y obedecen no a planes pre-establecidos sino a políticas presupuestales. Los agentes interventores no tienen claridad de las etapas y de su función en cada una de ellas: generalmente asumen roles que no les corresponden y con ello contribuyen al desmoronamiento de los proyectos.

Para entender un poco estos fenómenos, se puede hacer un acercamiento a la percepción que 
se tiene de estos procesos por parte de los emprendedores y los agentes externos.

En el caso de COROLAS se tiene un agente externo representado en la Precooperativa Multiactiva de Recicladores de la ciudad de Medellín (RECIMED), creada por iniciativa de la Secretaria del Medio Ambiente del municipio, la cual actúa en nombre del agente gubernamental. Aal respecto la representante legal de COROLAS, Miriam Rocío Puerta (2009) explica que:

RECIMED ha hecho una propuesta muy interesante a la Corporación Corolas y es tratar de implementar mecanismos para que ella logre su autosostenibilidad, que ella más adelante pueda ser autosostenible, que pueda contratar de manera autónoma con el municipio, pero primero se le tienen que dar las herramientas; por eso se le hace este acompañamiento técnico, administrativo y social.

También se deja ver en los testimonios que en el ejercicio de intervención ha habido problemas: Óscar Pérez Zapata (2009), de COOPEUNO dice:

(...) en sí, la cooperativa no ha tenido una autonomía total, porque siempre ha dependido de lo que hagan la secretaría de Desarrollo Social y UNIMINUTO. En estos momentos estamos comprendiendo que si no hay una autonomía total de estos entes, jamás podrá llegar la cooperativa a ser lo que nosotros queremos en la comuna: que sea, el motor del desarrollo de la economía solidaria.

Desde la Corporación Primavera, la asesora Yaneth Restrepo (2009) afirma que:s

... la autonomía en los procesos sociales es necesaria, porque lo que uno intenta con los asociados es que sean sujetos y no objetos de la intervención, pero también hay un momento, que aunque pueda tener autonomía, no quiere decir que se le quite el respaldo. Hay un grado de autonomía que es necesario.

En este mismo sentido, la versión de Efrén Bustamante (2009), desde el Circuito económico solidario de San Antonio de Prado, es para tener en cuenta:

El municipio debería hacer un diagnostico para ver qué tipo de capacitación necesita la gente, la cantidad de maquinarias que hay almacenadas es grande, el Presupuesto Participativo hay que revaluarlo, hay que hacer un debate abierto, no hay acompañamiento asignan dinero a proyectos sin estructura de mercadeo; a nosotros nos colocan como modelos porque hemos sacado esto solos adelante.

Puede decirse que la autonomía es uno de los principios y métodos más débiles en estas prácticas de emprendimiento: las encuestas dan cuenta de ello; el 76,2\% de los encuestados ha señalado que la autonomía no es total que sólo se da parcialmente y un $9,5 \%$ de los entrevistados afirma que esta autonomía es poca.

Se observa que el tema de la autonomía es supremamente sensible en el desenvolvimiento de los emprendimientos. Ya se ha dicho que la pérdida de ella lleva necesariamente a la desaparición de la autogestión, y al disminuir esta también disminuye la productividad; por tanto, todo el esfuerzo inicial se pone en riesgo y la sostenibilidad y permanencia ya no se aseguran.

Al respecto resultan válidas las apreciaciones de Razeto (1993, p.31) cuando reflexiona sobre las características que identifican a los emprendimientos de inclusión social:

Son organizaciones que quieren ser participativas, democráticas, autogestionarias y autónomas, en el sentido de que el grupo de sus integrantes se considera como el único llamado a tomar decisiones sobre lo que se hace, derecho que resulta del esfuerzo y del trabajo que cada uno y el grupo en su conjunto realizan.

Aunque de hecho los grupos tengan que experimentar varias formas de dependencia y sujeción frente a sujetos externos, lo que resalta su diferenciación es que las decisiones deben ser, de un modo u otro, legitimadas en el interior del grupo. 
Igualmente, estas prácticas emprendedoras pueden leerse a la luz del enfoque de justicia, equidad y desarrollo de Sen (2000, p. 345) que centra la atención en las libertades fundamentales y en la agencia y el criterio de los individuos, los cuales son responsables de su propio bienestar y los que deciden cómo utilizar sus capacidades, que están en relación de dependencia con la naturaleza de las instituciones sociales que pueden ser esenciales para las libertades individuales.

\subsection{Autogestión, participación y productividad en los emprendimientos solidarios de Medellín}

El método de la autogestión debe ser la principal característica del desenvolvimiento de un emprendimiento solidario; es lo que determina esta acción empresarial en el tiempo. En la medida en que se pretende satisfacer la necesidad de ingresos o de acceso a bienes y servicios por parte de sus miembros, éstos adquieren el derecho de ejercer control sobre el proceso y logro del objeto social, por lo que la acción empresarial se efectúa con el concurso de todos. Gaiger (2003, p 229) afirma: "los emprendimientos económicos solidarios abarcan diversas modalidades de organización económica, originadas en la libre asociación de los trabajadores, con base en principios de autogestión, cooperación, eficiencia y viabilidad".

Algunos aspectos que deben ser analizados en profundidad en este punto son los que se refieren a los temas de la contribución económica, especialmente en términos del trabajo y la productividad; la participación en la estructura organizacional y la toma de decisiones.

\subsubsection{Esfuerzos económicos y productivos}

Al respecto de la contribución con esfuerzos económicos y energía laboral, se encuentran algunos casos ejemplares en los emprendimientos estudiados que muestran que en la medida en que es un proyecto productivo, los asociados deben aportar en el proceso de trabajo. En ARPA, dice Luis Hernando Marín (2009):

(...) los asociados juegan un papel muy importante, ya que de ellos depende mucho el fortalecimiento y el crecimiento de la empresa como tal. Nosotros somos 15 y los que están laborando actualmente, aparte de nosotros que tenemos el contrato, 5 ó 6 personas más; pero hay personas que se han retirado, porque no tienen mucho conocimiento del cuento o creen que al estar dentro de una organización se van a tapar de plata, o creen que es que van a entrar allá y de una van a empezar a trabajar y no es así; pero también hay gente que sí comprende la lógica, o sea, de una u otra manera están ahí y a la hora que se les necesite responden.

Los emprendedores saben muy bien que lo único que poseen es su energía laboral. No cuentan con recursos económicos ni con medios materiales que aportar; apenas cuentan con algunos conocimientos específicos. Saben muy bien que deben perfeccionarse en el oficio y deben aportar a la producción. Por tanto, lo que ponen en común es su energía que es lo que llaman "sentido de pertenencia", y su capacidad de trabajo en cooperación. De ello dan cuenta las encuestas: las acciones de cooperación (en ocasiones o con frecuencia) se dan en casi un $86 \%$ de los casos.

Esta situación puede ser sustentada desde los aportes de Razeto (1998, p. 41-42) quien encuentra en los mencionados emprendimientos un elemento determinante en el factor trabajo, en el cual dice "se expresa la libre voluntad de una obra común... un trabajo que gratifica a las personas porque trabajan unidas generando vínculos de amistad, solidaridad, comunidad".

Pero no todo es disposición. El esfuerzo autogestionario conlleva a resistencias, sobre todo cuando se viene de experiencias laborales absolutamente dependientes o absolutamente independientes. El cambio en la cultura de la percepción de la remuneración, los horarios, la responsabilidad y las exigencias disciplinarias resultan, a veces, bastante 
traumáticas. Dice Luis Hernando Marín (2009) de ARPA, que: "eso más que todo pasa cuando las personas no tienen el conocimiento o no saben para donde van todavía, porque si uno sabe para donde va y hacia donde está direccionado, no le importa a uno trabajar más de las 8 horas, no le importa si es de los primeros que llega o es de los últimos que sale".

Es decir, la autoayuda resulta ser una actitud primordial en el emprendimiento. Pero también el esfuerzo económico (representativo del self help) es un claro símbolo de la autogestión y un mecanismo de exigencia de responsabilidad por el emprendimiento, haciendo entender que éste no puede depender económicamente de los agentes externos, ya que ello desvirtuaría toda la posibilidad de independencia. Alexander Nieto (2009), de ARPSE, explica cómo se produce este esfuerzo:

(...) esto se tiene por estatuto. Se arranca en el 2005 con la cuota de 1500 pesos mensuales para sostenimiento de la organización, con un incremento del 15 por ciento anual; hoy estamos pagando 4000 pesos mensualmente, para ese sostenimiento. Fuera de eso hay otras alternativas donde, por ejemplo, cuando un contrato por prestación de servicios, en el caso de la recreación, el socio hace la contratación y se le exige que le pague una pequeña cuantía a la organización que fue quien hizo el contacto.

Así, desde el punto de vista del aporte a la producción, la autoayuda, manifestación primaria de la autogestión, tiene que presentarse en el proceso productivo mismo. Gaiger (2003, p. 229) señala:

(...) los individuos excluidos del mercado de trabajo, o motivados por la fuerza de sus convicciones, y en búsqueda de alternativas colectivas de supervivencia, encuentran una alternativa en los emprendimientos económicos solidarios donde llevan a cabo actividades en los sectores de la producción o de la oferta de servicios comercialización y crédito. Se presentan en forma de grupos de producción, asociaciones, cooperativas y empresas de auto- gestión y combinan sus actividades económicas con acciones de índole educativa y cultural, valorando el sentido de la comunidad de trabajo y el compromiso con la colectividad social en la cual se insertan.

El garantizar la continuidad en la producción es una preocupación de todos los emprendedores cuando tienen sentido de pertenencia y a veces ellos mismos son los que realizan los contactos (una especie de mercadeo del emprendimiento), pero siempre -en una primera etapa- resulta difícil mantener la continuidad productiva. Entre los encuestados se afirma que sólo en un 4,8\% se logra siempre la continuidad en la producción y el 95,2\% afirman que sólo se da esta continuidad algunas veces, de manera esporádica.

Sin embargo, puede decirse que no se observan fuertes resistencias a la contribución económica para la organización y que la ayuda propia, el selp help, es básico en la sostenibilidad de la empresa social.

\subsubsection{La participación}

La autogestión no sólo es autoayuda, es, principalmente, una acción mediante la cual los agrupamientos humanos se auto-dirigen para lograr el objetivo previsto por el mismo grupo. Hay autogestión cuando los integrantes del emprendimiento se responsabilizan de la dirección y desenvolvimiento de su propia empresa. Pero para hacer autogestión es necesario que los emprendedores participen real y permanentemente en la toma de decisiones, en la planificación, la organización, la dirección, la ejecución y el control de la empresa.

En el grupo de encuestados, extraordinariamente, el $81 \%$ manifiesta tener mucha participación en las actividades del emprendimiento, lo que significa que su percepción es que de ello depende el desenvolvimiento: sólo si se participa, sólo si cada uno participa del emprendimiento, podrá haber satisfacción y éxito. 
Sin embargo, hay que señalar que la autogestión no es un ejercicio exclusivamente de participación económica, de aporte de energía de trabajo, de acción productiva y de disposición a estar en los diferentes escenarios del emprendimiento. Más allá, se trata también de un método por el cual se aprende a vivir y a decidir en democracia y ésta tiene validez en la medida en que los emprendedores participen de los espacios para la toma de decisiones.

La participación en la toma de decisiones es la reafirmación de la autonomía. Si no se establecen estos espacios de participación, el agente externo tomará las decisiones o, en su defecto, lo hará el líder o el simple administrador aislado.

En este aspecto, ante la pregunta acerca de su participación en la toma de decisiones, se descubre en las respuestas una debilidad en los emprendimientos locales estudiados. El 51,4\% de los encuestados indican que no tienen o tienen muy poca participación; sólo un 23,8\% aseguran tener control sobre muchas o todas las decisiones.

En el caso de ARPA Luis Hernando Marín (2009) comenta:

... solamente funciona la asamblea y la junta directiva, o de pronto cuando hay reunión extraordinaria se convoca a todos, básicamente es eso, no tenemos comités o cosas así, pero la idea si es ir pensado como en tener acercamientos con los otros líderes comunitarios, ya que esto ha sido una gran dificultad.

En ARPSE, Alexander Nieto (2009) explica que los espacios de participación son:

(...) la asamblea general; no se tienen organizados unos comités sino unas coordinaciones de proyectos; de igual forma se tienen otros estamentos, las integraciones que se hacen, las capacitaciones de múltiples organizaciones que llegan a trabajar con nosotros, todo eso conlleva a que estemos unidos en lazos casi afectivos dentro de la organización y que participen ante todo. La asamblea general se convoca con veinte días de anticipación, se les hace telefónicamente, por escrito, se coloca en cartelera, se les da a conocer la fecha de la asamblea y se hace lo posible por que todos estén dentro de esa asamblea. La participación en la asamblea es del 98\%, digámoslo del 90 por ciento, pues tenemos socios por actividades externas, en lo que ellos hacen, por ejemplo en la última asamblea nos faltaron 2 asociados, no llegaron, pero de igual nos dieron a conocer que se sometían a las decisiones tomadas en esta asamblea.

Otros ejercicios de participación en ARPSE tienen que ver con la educación y la integración, Al respecto Alexander Nieto (2009) argumenta:

En la asociación todos los meses tenemos ejercicios de sensibilización y capacitación en áreas del fortalecimiento de la organización, y se hacen actividades de integración, que muchas veces se hacen dentro de las mismas actividades que se están realizando. Caso puntual se forma un convite para una llena de bolsas en el vivero y todo se hace alrededor de un sancocho que patrocina la organización, y todo eso conlleva a la integración.

Con relación al relato de estas experiencias de participación, se hacen pertinentes los aportes de Davis (2001) quien dice que en la empresa social la gente debe ser primero y principalmente alentada para actuar en conjunto, lo que constituye la esencia de la asociación, porque si se quiere el éxito empresarial es necesario que los miembros se unan y comprometan en una comunidad económica y social. Sólo en la medida en que haya participación en los espacios de toma de decisiones se asegurará la autogestión en su máximo nivel y se mantendrá la autonomía.

\subsubsection{La productividad}

Ahora bien, siguiendo las características del Factor Comunidad, explicado por Razeto (1998) como factor de productividad en la racionalidad de la economía solidaria, el liderazgo es una condición sine qua non para alcanzar la productividad dentro de 
un emprendimiento solidario. La participación que implica la opinión de todos, el establecimiento de acuerdos y consensos, las posibilidades de aportar al mejor desempeño organizativo o el acceso a la educación y a la integración, resulta ser un mecanismo motivador para la productividad.

Este es un concepto que está relacionado con la capacidad competitiva de las empresas. Para la empresa social resulta ser una categoría inmanente a su naturaleza, ya que está relacionado con dos situaciones especiales: de un lado, la posibilidad que tienen estas empresas de maximizar factores productivos que han estado ocultos (invisibilizados) y que son los que dinamizan su proceso; del otro, la necesidad de extender este proceso para obtener resultados económicos. Si se utiliza un mismo volumen de recursos aumentando los factores ocultos, resulta de ello una mayor rentabilidad y una mayor disposición del valor agregado para ser redistribuido y cumplir la función social.

Siguiendo las tesis de Chirinos y Chinchilla (2003), investigadores venezolanos, en las cooperativas (como ejemplo de empresa social) los trabajadores deben estar dispuestos a optimizar la productividad por la relación clara que existe entre esta y su bienestar, en tanto son dueños de los medios de producción y responsables de la obtención y transformación de los mismos. Luis Hernando Marín (2009) de ARPA sostiene que:

(...) cada uno de los asociados aporta con su trabajo buscando formas de sostenimiento para la misma organización, que no solamente sea trabajar con el municipio de Medellín, sino que nos podamos abrir a otro público diferente, que requiera nuestros servicios. Entre ellos está este vivero, yo soy el encargado, que de una u otra forma ha aportado capital para la asociación para seguir adelante y al desarrollo productivo, porque esto es otro negocio de la asociación.

En este sentido puede advertirse también que los encuestados, en un $100 \%$ consideran que los logros del emprendimiento en términos de la productividad, hasta el momento, son buenos; en igual porcentaje se consideran satisfechos con el proceso del emprendimiento.

\subsection{Resultado social producido por los emprendimien- tos sociales y solidarios de Medellín}

La razón de existencia de una empresa social es la producción de efectos sociales, que generen transformaciones en la vida de los sujetos participantes. Ellos constituyen el mejor indicador de desempeño y de logro del objeto social. Los resultados sociales son los logros alcanzados en cuanto a cumplimiento de objetivos propuestos respecto de mejorar el nivel de vida de la gente y el indicador de mejoramiento constituye el impacto social.

Un emprendimiento solidario se entenderá exitoso cuando alcance un cierto grado de cumplimiento de su política y estrategias sociales.

Cuando se evalúa el impacto y comparte esta evaluación, se logra también un nivel más elevado de empoderamiento del emprendimiento, porque ello ha significado la producción de capitales sociales que lo hacen sostenible.

En resumen, las diferentes opiniones respecto de los ejercicios productivos solidarios coinciden en señalar que estos tienen sentido cuando en su evaluación se descubre un efecto positivo de cambio en las condiciones de vida de los emprendedores y la comunidad local.

Sobre este aspecto en el que los emprendimientos urbanos, así como las asociaciones o grupos comunitarios, desarrollan sus actividades en el barrio donde generan una gran capacidad de respuesta creativa y de resistencia para salirle al paso a los problemas económicos y de exclusión social, resultan contextualizadoras nuevamente las palabras de Razeto (1993, p. 29): "el mundo popular, puesto a hacer economía, la hace a su modo, con sus valores, con sus modos de pensar, de sentir, de relacionarse y de actuar".

En el caso de la muestra de emprendimientos estudiados para efecto de esta investigación, los in- 
dicadores base manifiestan una serie de situaciones particulares en sus emprendedores: se encuentran ubicados en lugares de alta marginalidad económico-social (corregimientos y zona nororiental de Medellín), son personas de estratos económicos 1 y 2, su escolaridad (en un 38\%) es de nivel básico primaria o inferior.

Se tiene también que los emprendedores en un $57 \%$ son adultos entre los 26 y 41 años, lo que denota responsabilidades familiares de primer orden.

De modo que se infiere que los emprendedores son personas con altos índices de necesidades básicas insatisfechas y se unen al proceso empresarial para tratar de mejorar esas condiciones mediante acceso a dos satisfactores primarios, el ingreso por el trabajo y el elevamiento de la auto-estima.

Varios comentarios de María del Carmen Jiménez (2009) de la Corporación Primavera de Medellín denotan un primer nivel de acercamiento al resultado o efecto personal y comunitario:

Yo he crecido como persona, he entendido el propósito de la Corporación. La Corporación tiene un gran reconocimiento a nivel del sector, porque lleva muchos años... se ha podido ayudar, a través del jardín infantil, porque los niños y niñas son de este sector. Ya se reconoce el trabajo de la institución. En muchas instituciones de la ciudad ya la conocen.

Carmen Janet Rojas (2009), una de las beneficiarias de la Corporación Primavera, dice que su calidad de vida ha mejorado porque "el trabajo en la entidad lo hace sentir a uno vivo, sentirse con seguridad, que uno puede hacer las cosas por uno mismo, valorarse más y que uno puede no depender de las otras personas, que uno puede trabajar".

Se destacan también aspectos sobre el impacto en la comunidad desde el mejoramiento del entorno y el medioambiente. Luis Hernando Marín (2009), de ARPA, considera que su trabajo ha permitido construir una mejor percepción de la realidad:

Porque yo no tenía conocimiento de la importancia de trabajar por el medio ambiente. Ahora que estoy metido en el tema de lo ambiental me parece que es algo muy importante, porque me parece que todos estos materiales que uno recoge evitan de cierta manera que lleguen al relleno sanitario, y nos permite a nosotros darle un mejor aprovechamiento; además Usted va a cualquier casa del corregimiento y le dice, vengo por la materia orgánica, y saben reciclar.

Myriam Rocío Puerta (2009), de COROLAS, también aporta unas ideas para entender los logros de un emprendimiento social. Entiende que hay una relación entre la comunidad y el emprendimiento:

La comunidad nos da el orgánico, entonces se purifica el ambiente, se descontamina, los rellenos sanitarios van a tener más vida útil. Como corregimiento estamos siendo los pioneros. Otro proyecto que nosotros hemos manejado ha sido el de la culinaria nativa, o sea, es que la gente vota los alimentos, la gente no sabe que a cocina natural que consiste en el mejor aprovechamiento de productos de la tierra, es aprender que de las cáscaras se preparan una cantidad de alimentos: sopas, pudín, tortas; nosotros tuvimos la oportunidad de dar unos talleres y clases en ese aspecto y mejoramos la calidad de vida. Fueron maravillosos esos talleres que realizamos y precisamente lo hicimos con presupuesto participativo, pensamos que la plata que nos ganamos no va a ser para ejecutarla aquí al interior, va a ser para ejecutarla con la gente del corregimiento en esas capacitaciones, eso va a conllevar a mejorar la calidad de vida de las personas, saber aprovechar al máximo los alimentos, el espacio, el aire, la naturaleza.

De otra parte tener tranquilidad cuando se perciben ingresos es algo fundamental en la vida y estos emprendimientos garantizan en parte esa posibilidad:

Elkin Álvarez (2009), otro asociado de ARPA, argumenta que su vida ha mejorado: "(...) porque uno se siente muy bien cuando le pagan... hay una satisfacción que me agrada ver a mis hijas bien, al menos hay dinero para sustentos, para un 
viático, para que tengan como tomarse un algo en el colegio".

Manuel David González (2009) de COOPEUNO, explica alrededor del tema del mejoramiento de la calidad de vida que:

Yo lo podría decir muy optimistamente, podría ser un 100 por ciento, y yo le diría que así es. Vea hasta me pongo "arrosudo", porque hemos visto los resultados, hemos visto estas unidades productivas avanzar, su gente que está participando se están beneficiando, han mejorado la calidad de vida de su familias, que es uno de los objetivos.

Al tabularse las preguntas relacionadas con los resultados del emprendimiento, desde el punto de vista del crecimiento personal, los encuestados manifiestan unas respuestas bastante positivas. En el 100\% responden que han mejorado sus condiciones de vida, sobre todo porque el emprendimiento les posibilita el acceso a un empleo (en un 90,4\%) o porque les permite el acceso a bienes y servicios (en un $4,8 \%$ ) o porque les asegura ingresos (en un $4,8 \%)$.

Al respecto también es diciente la versión del señor Efrén Bustamante (2009), líder del Circuito Económico de San Antonio de Prado:

En el circuito se generan los siguientes empleos:

Estampación 10 personas; Ecotejidos 7 personas, Serviteca 6 personas, más indirectos; Bisutería más o menos 6 y Creaciones Ego más o menos 12 directos y los indirectos varían de 18 a 30. En el circuito se ha cualificado su liderazgo, ha influido para que otros alrededor nuestro se preocupen por generar proyectos productivos, gente que ha obtenido un sustento provocando mejor calidad de vida, lo más importante que queremos es consolidarnos como empresa y que sean los asociados quienes trabajan en área administrativa productiva y contable convirtiéndose en proyectos asociativos verdaderos donde la gente tenga pertenencia.

En todo caso, para los encuestados, la empresa social es el mecanismo de inclusión social-desde su percepción-, por el cual, a través del trabajo, se mejoran las condiciones de vida, se perciben ingresos y se contribuye a acceder a bienes y servicios que satisfacen varias de sus carencias primarias. Es por esta razón fundamental que se hace imprescindible dar continuidad al emprendimiento (desde el punto de la estabilidad en los puestos de trabajo en la producción misma), porque de no hacerse volverá al emprendedor a las filas de la exclusión social y económica.

\subsection{Redes e intercooperación en emprendimientos solidarios de Medellín}

Mediante la intercooperación, dice Fauquet, (1973, p. 56) "los cooperadores de ciudades y de aldeas se hacen cargo de los lazos que los ligan. Entre unos y otros se establece una comunidad de sentimientos que tiende a hacer prevalecer en sus relaciones las normas de una equitativa solidaridad, el respeto recíproco de sus condiciones de vida y trabajo".

Los emprendimientos solidarios, para ser eficaces y alcanzar el éxito, deben entender que necesitan de actuar en conjunción con otros emprendimientos, a través de conexiones, alianzas o compartimiento de soluciones y experiencias.

Siguiendo la tesis de Moreno (1990, p. 63) la palabra interacción puede explicar también este fenómeno de relacionamiento entre las partes ya que es "una forma universal de nexos entre cuerpos y fenómenos que se expresa en la influencia de unos sobre los otros o frente a los otros y en las modificaciones que sufren por esas mismas relaciones".

Argumenta Moreno (1990) que histórica y biológicamente está probado que organismos iguales, con características genéticas semejantes, son sujetos de actitudes y procesos de integración. Esto significa que es un estado natural de los seres vivos y explica la tendencia humana a facilitar su acercamiento mediante la identificación de intereses comunes, económicos y sociales. Pero son los pensadores asociacionistas, cooperativistas y socialistas

Semestre Económico, volumen 14, N28, pp. 77-94 • ISSN 0120-6346, enero-junio de 2011, Medellín, Colombia 91 
quienes superan esta concepción organicista del concepto para hacerlo más propio de la conciencia humana: al proponer la federación se establecen principios de soberanía y autonomía, ejercicio de derechos, cumplimiento de responsabilidades, arreglo de diferencias y búsqueda de la prosperidad común.

En el contexto de los emprendimientos estudiados, dice Manuel David González (2009), del Consejo de Administración de COOPEUNO que:

El objetivo y la misión en un principio fue crear una red que estuviera generando empleo en nuestra comuna e integrando ciertas personas, como también ciertas organizaciones, como son entidades productivas, en ese momento nosotros hablamos también de agrupamientos, que en la comuna han existido. La intención... era generar un empleo digno mediante la comercialización de los productos que se hacen en nuestra comuna, o sea ser el eje central de todas estas organizaciones que estaban sueltas, aunándolas, volviéndonos una cooperativa para poder generar esto.

Este proyecto en particular, tiene la virtud de integrarse a procesos amplios de organización comunitaria y de desarrollo de la ciudad... ahora están reunidos nuestros otros compañeros en la Corporación Convivamos, porque está que arranca ahora de nuevo el plan de desarrollo en nuestra comuna. Y vea, fuimos pioneros de los planes locales de desarrollo en la ciudad de Medellín.

Se considera que el tema de la integración y las redes es de importante tratamiento en los emprendimientos de Medellín, teniendo en cuenta que ante la pregunta que al respecto se hizo a los encuestados, en una tercera parte éstos afirmaron que no existen estas acciones y un 38\% verifican que se da sólo en algunas ocasiones. Puede decirse que no es de la cultura de los emprendimientos de Medellín (aunque en la política pública se ha formulado así) el desarrollo amplio de relaciones de intercambio, de intercooperación o de formación de redes.

\section{CONCLUSIONES}

A partir de la información expuesta en términos de la lectura de referentes conceptuales y teóricos, y de la interpretación y análisis de fuentes provenientes del referente experiencial, puede concluirse que las condiciones que se identificaron como relevantes para el éxito de los emprendimientos solidarios en Medellín están dados por

1) El nivel de acercamiento al cumplimiento de su objeto social. Un emprendimiento social se debe regir por un espíritu que trasciende lo estrictamente económico, colocando la iniciativa empresarial al servicio del proyecto grupal o comunitario.

2) El establecimiento en un territorio concreto, que se constituye como un nuevo componente de la cultura de sus gentes, con noción de pertenencia a la localidad.

Los emprendimientos observados tienen un firme asiento local. Se construye alrededor de un barrio un conjunto de barrios (identificados cultural y económicamente) o una determinada zona rural (corregimientos). Dichos emprendimientos, aunque actúan en el territorio de la ciudad y son influenciados por las condiciones de este territorio, son esfuerzos mucho más localizados y no presentan pretensiones de expansión, por lo menos en la etapa en la cual se encuentran. Seguramente si se mantiene ese criterio de endogenismo local habrá mayor posibilidad de permanencia, sostenibilidad y éxito.

3) El desarrollo de procesos de encadenamientos de empresas de su mismo tipo que tienen semejanzas y necesidades comunes, logra producir mejores niveles de cooperación; aunque todavía no avanzan de manera decidida a construir integraciones y hacer acuerdos de intercooperación para responder a diversas necesidades de su desenvolvimiento, puede decirse que todos los emprendimientos han nacido del deseo de construir redes locales y algunos de ellos son estrictamente una red de pequeños emprendimientos. 
4) El fomento y fortalecimiento de condiciones de autogestión y participación, no simplemente como un ejercicio actitudinal o de aceptación de una doctrina empresarial sino como un asunto de mayor incorporación en sus prácticas, buscando asegurarle a la gente continuidad en el trabajo, ingresos y participación en toma de decisiones.

Finalmente, en cuanto al papel de los agentes externos, y concretamente desde el rol del gobierno local, se considera recomendable pensar en una política pública que contemple o agregue una especie de metodología de intervención, ya que la acción promotora tiene unas fases que es necesario de seguir hasta su último momento.

\section{BIBLIOGRAFÍA}

Alcaldía de Medellín (2004). Plan de Desarrollo 2004-2007: Medellín compromiso de toda la ciudadanía. [En línea] Veeduría de Medellín, 188p. Disponible en http:// www.veeduriamedellin.org.co/plan _ desarrollo2. shtml? $\mathrm{x}=278$ [Consultado marzo de 2009].

Alcaldía de Medellín (2008). Plan de Desarrollo 2008-2011: Medellín es solidaria y competitiva. [En línea] Alcaldía de Medellín, 236p. Disponible en http://www.medellin. gov.co/alcaldia/jsp/modulos/P_ desarrollo/P_desarrollo.jsp?idPagina $=380$. [Consultado marzo de 2009]

Álvarez Valencia, Hugo Andrés (2008). Economía solidaria. Análisis del concepto. Tesis para optar al grado de licenciado en ciencias socioeconómicas. Universidad de Valparaíso. Facultad de Ciencias Económicas y Administrativas. Carrera de Licenciatura en Ciencias Socioeconómicas.

Álvarez, Elkin Rodrigo (2009). Asociación de recuperadores pioneros de AltaVista (ARPA). Entrevista inédita. Corregimiento de AltaVista, Medellín.

Bustamante, Efrén (2009). Circuito económico solidario de San Antonio de Prado. Entrevista inédita. Municipio de Itagüí.

Congreso de Colombia. Ley 1014 de Fomento a la Cultura del Emprendimiento. Diario Oficial No. 46.164, del 26 de enero de 2006. Bogotá D. C.
Congreso de Colombia. Ley 454 de Economía Solidaria. Diario Oficial No. 43.357, de 6 de agosto de 1998. Bogotá D. C.

Chirinos Zárraga, Emilio y CHINCHILLA, Haydeé C. (2003). Productividad y empresas cooperativas. [En línea] Revista Venezolana de Ciencias Sociales. Vol.7. No 1, Maracaibo-Estado de Zulia-Venezuela, UNERMB, enero de 2003. Disponible en: http://200.11.182.194/ investigacion/index.php?option=com _ contentEv iew $=$ articleEid $=18$ Eltemid $=27$. [Consultado marzo de 2009].

Davis, Peter (2001). Propósitos, valores y administración cooperativa en el siglo XXI. [En línea] El cooperativismo en tiempos de cambio: un proyecto innovador, una ley integradora. Montevideo, Neticoop, junio de 2001. Disponible en: http://www.neticoop.org.uy/ article207.html, [Consultado febrero de 2009].

DNP - Departamento Nacional de Planeación- (2007). CONPES No 3484. Política nacional para la transformación productiva y la promoción de las micro, pequeñas y medianas empresas: un esfuerzo público privado. [En línea] Consejo Nacional de Política Económica y Social. República de Colombia. Ministerio de Comercio, Industria y Turismo, Departamento Nacional de Planeación. Bogotá, agosto de 2007. Disponible en: http://www.snc.gov.co/Es/Politica/Documents/ Conpes\%203484.pdf. [Consultado abril de 2009].

Fantova, Fernando (1995). Tercer sector e intervención social. Madrid: PPC, 2005. Instituto Nacional de Empleo (INEM). Plan Nacional de Valorización. Madrid: INEM. $138 \mathrm{p}$.

Fauquet, Georges (1973). El sector cooperativo. Buenos Aires: Intercoop. $177 \mathrm{p}$.

Gaiger, Luis Inácio (2003). Emprendimientos económicos solidarios, p. 131-143. En: CATANI, Antonio David (Organizador): A outra economía. [En línea] Veraz Editores/Unitrabalho, Porto Alegre. p. 229-241.Disponible en http:// www.urbared.ungs.edu.ar/textos/ emprendimientos. [Consultado en junio de 2008].

Gallo Camacho, Jaime (2005). Las empresas sociales y sus factores de éxito. [En línea] Líder Empresarial Añoll, No 129, Aguas Calientes-México, septiembre de 2005. Disponible en http://www.tau.org.ar/upload/ 89f0c2b656ca02ff45ef6la4f2e5bf24/emprendimien- 
tos econ micos solidarios.pdf. [Consultado agosto de 2009]

Gide, Charles (1968) Cooperativismo. Bogotá: Publi-coop, $206 \mathrm{p}$.

González, Manuel David. (2009) Precooperativa Multiactiva COOPEUNO. Entrevista inédita. Medellín.

Guerra, Pablo (2006). La economía de la solidaridad. Umbrales No 168, Montevideo- Uruguay, Julio 28 de 2008.

Jiménez, María del Carmen (2009). Corporación Primavera. Entrevista inédita. Medellín.

Lopera García, Luz Dolly y Aguirre Echavarría, Francisco (2008). Caracterización socioeconómica y organizacional de los emprendimientos asociativos desarrollados en la ciudad de Medellín 2005-2007. Medellín: FUNLAM.

Marín, Luis Hernando (2009). Asociación de recuperadores pioneros de AltaVista ARPA. Entrevista inédita. Corregimiento de AltaVista, Medellín.

Maxneef, Manfred; Elizalde, Antonio y Hopenhayn, Martín (1995). Desarrollo a escala humana una opción para el futuro. Suecia: Cepaur. Fundación Dag Hammarskjold. 94p.

Moreno Avendaño, José del Carmen (1990). Cooperativismo años 80. Patología de una crisis. Bogotá: Guía. 159p.

Nieto, Alexander (2009). Asociación de Recuperadores prestadores de servicios de Santa Elena. Entrevista inédita. Corregimiento de Santa Elena, Medellín.
Ortiz Roca, Humberto (2005). Hacia la solidaridad en la economía. [En línea] Vinculando: Revista electrónica sobre desarrollo sustentable, México, 2005 Disponible en: http://vinculando.org/economia _ solidaria/ humanizar_economia _ global/solidaridad_economia _ 4.html. [Consultado agosto de 2008].

Pérez, Oscar (2009). Precooperativa Multiactiva COOPEUNO. Entrevista inédita. Medellín.

Puerta, Miriam Rocío (2009). Corporación Olas COROLAS. Entrevista inédita. Corregimiento de San Antonio de Prado. Medellín.

Razeto Migliaro, Luis (1993). Los caminos de la economía de solidaridad. Santiago: Vivarium.183p.

Razeto Migliaro, Luis (1998). Alternatividad y racionalidad de la economía solidaria. Medellín: Escuela Colombiana de Economía Solidaria. 59p.

Razeto Migliaro, Luis. (1992). Fundamentos de la teoría económica comprensiva. Santiago de Chile: Ed. PET, 506p.

Restrepo, Yaneth (2009). Corporación Primavera. Entrevista inédita. Medellín.

Rojas, Carmen (2009). Corporación Primavera. Entrevista inédita. Medellín.

Sen, Amartya (2000). Desarrollo y libertad. Barcelona: Editorial Planeta, 440p. 